\title{
Seizure Management in the Intensive Care Unit
}

\section{Jane G. Boggs, MD, FACNS, FAES ${ }^{1, *}$}

\author{
Address \\ ${ }^{*}, 1$ Comprehensive Epilepsy Center, Wake Forest University, Winston-Salem, NC, USA \\ Email: jaboggs@wakehealth.edu \\ Published online: 21 0ctober 2021 \\ (๑) The Author(s), under exclusive licence to Springer Science+Business Media, LLC, part of Springer Nature 2021
}

This article is part of the Topical Collection on Epilepsy

Keywords Intensive care unit - Seizures - Status epilepticus - Continuous electroencephalogram · Antiepilepsy drug · Intravenous anesthetic therapy

\begin{abstract}
Purpose of this review This review presents current therapy for seizures in the intensive care unit. The reader is provided with recent evidence regarding the use of EEG in determining treatment for acute seizures. Proposed treatment approaches for seizures and status epilepticus are provided. Controversies and complexity of selecting treatments are discussed.

Recent findings Critical Care EEG Monitoring Research Consortium analyzed the association of periodic and rhythmic electroencephalographic patterns with seizures and found that lateralized and generalized periodic discharges and lateralized rhythmic delta were associated with increased seizure risk. Applications using modified EEG techniques have demonstrated more rapid feedback to the ICU than was previously possible.

Summary Accurate diagnosis and efficient treatment of seizures in the ICU is challenging due to patient factors, complexities of antiepileptic drug therapy, and the required expertise for EEG interpretation. Selection of optimally effective therapy for seizures or status epilepticus depends on multiple factors, making collaboration between neurophysiologists and the ICU team of paramount importance.
\end{abstract}




\section{Introduction}

The incidence of seizures in intensive care units (ICU) varies based on underlying diagnosis and is reported at 3.3-34\% [1]. Determining the approach to isolated or multiple seizures depends on reported history and clinical assessment as well as electroencephalogram (EEG) and supportive testing. Knowledge of any prior history of seizures, epilepsy, and prior anti-epilepsy drugs (AED) is often helpful, but may be a luxury unavailable to the ICU team. In the absence of confirmed history or EEG evidence, the events leading to admission cannot always definitively be characterized as epileptic. Patients in the ICU are often intubated and sedated, so history, when available, must be sought from caregivers, observers, and the electronic medical records (EMR). One of the challenges of current medical care is failure of communication among different EMRs. If incorrectly suspected seizure activity must be assumed to warrant ongoing AED, not only is unnecessary medical care possible, but will also result in deterioration of the neurologic exam and medical complications of such therapy. This review will focus on the various scenarios in which patients can have seizures or seizure-like activity in the ICU, as well as proposing approaches to management. Status epilepticus (SE) is the most extreme manifestation of seizures in the ICU and has been reviewed extensively in other publications[2-5]. This review will summarize diagnostic and treatment concerns of SE which are specific to the ICU.

As with any complex medical condition the most important element in a correct diagnosis is a careful history. Reported "seizures" without any description should prompt a search for more detail. Observations of the situation preceding or triggering the ictus and the post-ictal state should be reviewed. Useful descriptive characteristics include behavioral changes, speech changes, focal increased or decreased tone, focal body and eye movements, lateral tongue bites, and duration of suspected seizures. In the acute postictal state, focal weakness, focal hyperreflexia, or aphasia may be present transiently after the seizure, suggesting an underlying cerebral lesion. MRI is the preferred imaging for correlation with these exam findings. High-resolution CT may provide information of large intraparenchymal abnormalities if MRI is impossible, but is otherwise often unrevealing [6]. If the patient is not returning toward the usual baseline after the clinical event, EEG can identify or rule out ongoing ictal activity. MRI compatible electrodes should be used if imaging is expected after EEG begins but these are more expensive and may require replacement sooner than standard EEG electrodes [7]. The typical short-term EEG of only 30 min may be too brief to identify patterns at risk for seizures, and thus longer recordings are preferable in comatose or patients at high risk for seizures.

Some patients may have convulsive tonic or clonic movements which are interpreted as seizure but in fact are not ictal. Nonepileptic events (NEE) in acutely ill patients are more likely medical than psychogenic and may be due to syncope, shivering, tremors, rigors, posturing, or dystonic movement disorders [8]. ICU patients less commonly have psychogenic NEE, usually conversion disorders rather than malingering, and these can be associated with a high medical utilization cost [9]. A 5-year review of admissions subsequently 
found to be NEE on continuous EEG (CEEG) determined that 15\% were intubated by emergency personnel prior to ICU admission [10]. It is also common for such aggressively treated NEE patients to receive doses of benzodiazepines, resulting in sedation, further impeding history and exam. Benzodiazepines prior to the EEG cause a marked increase in diffuse fast beta and suppression of amplitude so that diagnostic cerebral patterns cannot always be adequately seen. This can delay correct diagnosis further, and increase ICU length of stay.

All patients presenting with seizure-like activity should have serum laboratory analysis to identify potential metabolic causes. Hyperglycemic and hypoglycemic seizures may manifest as focal or generalized patterns both clinically and on EEG, despite the absence of underlying cerebral structural abnormality [11]. The presence of fever, leukocytosis, and systemic signs of infection should prompt consideration of further workup, including lumbar puncture. Seizures, however, can often cause elevations of white blood cell count and acute phase reactants, which will usually be less persistent than when caused by infection. Acute CNS infections can directly precipitate seizures due to cortical irritation, but systemic infections as well as antibiotics can also result in acute encephalopathy often with seizures. Acute altered mental status with fever and seizures should always prompt further investigation for a fulminant infectious process such as meningitis or encephalitis. While neurologic symptoms commonly occur in COVID-19 patients, the risk for seizures appears usually to be more directly related to the acute illness [12]. Ictal confusion and postictal states may be indistinguishable clinically, appearing as a waxing and waning encephalopathy [13]. EEG must be performed during these symptomatic periods to determine if altered mental status represents nonconvulsive seizures or nonconvulsive SE (NCSE).

Achieving a highly reliable recording of EEG in the electrically hostile environment of the ICU requires skilled EEG technologists, fastidious recording hookup and maintenance, as well as optimized EEG equipment [14]. EEGs should be performed at bedside according to the standards of the American Clinical Neurophysiology Society (ACNS) [15] and the American Society of Electrodiagnostic Technologists (ASET) [16]. There may need to be modifications of standard electrode placement due to the presence of wounds and craniotomy defects, cerebral pressure monitoring devices and drains as well as the inability to reposition patients. Application and removal of electrodes and testing protocols must be conducted with enhanced safety precautions for the technologist in patients with infections, such as COVID-19 [17]. Recordings are susceptible to electrical artifacts from ventilators, pumps, and other devices which can produce confounding periodic or rhythmic artifacts. Artifact intrudes not just from devices attached to the patient on EEG, but also from staff movement and equipment in adjacent patient spaces. Careful identification of sources of artifact requires skillful EEG technologists as well as experienced reviewing neurophysiologists [18]. There is a constant barrage of electrical noise as well as an abrupt biologic artifact from suctioning, repositioning, and procedures performed at bedside, so longer CEEGs are higher yield [19]. CEEG is usually recorded with video, which is helpful in correlating patient clinical events with EEG patterns, but only if the patient is visible on camera. This requires maintaining the position of the camera with an adequate view of the patient and ensuring staff, family, and equipment 
do not block the camera. Automated switching to infrared camera mode is necessary when lighting is decreased. CEEGs allow event marking, usually by a bedside trigger, which when activated results in integrated marks on the EEG recording. The setup of the CEEG at bedside should include education of ICU staff for use of this event marker or other methods of documentation of events. The technologist or ICU staff should perform a consistent daily stimulation protocol to assess cerebral reactivity of CEEG, one that is appropriately tailored to the patient's condition. Lack of reactivity or spontaneous variability for prolonged periods in the absence of sedation implies a worse prognosis for meaningful neurologic recovery. Specific stimulation protocols vary among institutions but should at minimum include auditory, tactile and passive eye opening and closure [20].

The gold standard in the diagnosis and characterization of seizures is EEG synchronously demonstrating ictal patterns with a video recording of typical clinical events. ICU patients may have varying degrees of somnolence or sedation, and others are treated with paralytics, often making clinical events less apparent on video. Nonconvulsive seizures are common in the ICU, with electrographic patterns appearing as rhythmic spike, sharp, or slow discharges that are distinct from the preceding background. Evolution, a typical characteristic of ictal EEGs, may or may not be apparent, but is highly consistent with seizure when it is seen. Usually accepted criteria for a discharge to be considered a seizure is a rhythmic epoch $>2 \mathrm{~Hz}$ for at least $10 \mathrm{~s}$ [21]. There are, however, many rhythmic patterns which are less "typical" which have been characterized as part of the "ictal-interictal continuum" (IIC) [22]. One such pattern is the brief rhythmic discharge (BRD) which is similar in appearance to longer electrographic seizures and has a high associated risk for the development of seizures [23]. The CEEG may decay following seizures into more protean periodic and rhythmic patterns, which can variably correlate with the risk for seizures. The Critical Care EEG Research Monitoring Consortium (CCERMC) of the ACNS found that lateralized periodic discharges, lateralized rhythmic delta, and generalized periodic discharges were associated with a risk for seizures, but generalized rhythmic delta activity alone had no unique association with seizures [24]. Patterns of the IIC may change rapidly over prolonged recordings, extending the potential recording of CEEG for many days. In the absence of medication effects on the EEG, unchanged patterns with no seizures for over $24 \mathrm{~h}$ indicate that the CEEG is not contributing to management decisions and can be discontinued. Validated machine learning models have been proposed incorporating clinical and EEG characteristics to predict risk for the development of seizures [25]. Implications of many rhythmic and periodic EEG patterns remain controversial and there may be disagreement between neurophysiologists whether patterns are ictal, a lowered threshold for seizure, or simply encephalopathic [26]. A benzodiazepine (BDZ) challenge can be performed at bedside when such patterns cannot be clearly identified as ictal [27]. After administration of a dose of intravenous $\mathrm{BDZ}$, the EEG is compared to pre-dose appearance. Improvement in both background and clinical function provides circumstantial evidence supporting that the pre-dose pattern was ictal. It must be remembered, however, that BDZs decrease the amplitude of the EEG and produce dense beta, which can 
be falsely interpreted as the resolution of a pattern of unclear significance, which may further obscure the EEG interpretation.

Patients with hypoxic encephalopathy commonly exhibit myoclonus with abrupt increased amplitude periodic bursts of myotonic activity on EEG. Clinical twitching may not be obvious, increasing the suspicion that the discharges are ictal. Back-averaging the EEG with EMG can show no preceding cortical waveform before the myoclonic potential [28] Alternatively, administration of a short-acting paralytic can result in the elimination of the myoclonic artifact, allowing only the cerebral waveforms to be visualized. If an underlying rhythmic or periodic pattern remains after confirmed paralysis, myoclonic discharges are of cortical origin. Cortical myoclonus, while not definitively ictal, is frequently stimulus sensitive (stimulus-induced rhythmic, periodic, or ictal discharges (SIRPIDS)) and may progress to nonconvulsive status epilepticus [29].

Critical care CEEG (CCEEG) describes a modification of CEEG recordings which include a graphic display of compressed EEG processed by amplitude, frequency, and rhythmicity trends over hours to days. CCEEG can allow rapid analysis, usually initially performed by neurodiagnostic technologists, of long intervals of multiple studies in a shorter period of time than required to review by standard methods [30]. Detailed analysis of corresponding raw CEEG is done by neurophysiologists for the entire study or just segments of interest. The consensus statements by the ACNS and Critical Care EEG Monitoring Research Consortium delineate the indications [31] as well as highly specific technical and personnel specifications [32] for CCEEG studies. Institutions with large volumes of simultaneous CEEG benefit from the efficient review possible with CCEEG, while those with lower volumes of diagnostic studies can manage patients effectively with CEEG review methods.

While CCEEG or traditional CEEG are optimal diagnostic modalities for seizures in the ICU, limitations of equipment and technologist availability result in an opportunity for supplemental technology. Devices which can be applied quickly at bedside by members of the ICU team, rather than EEG technologists, have been shown to have screening utility when CEEGs and skilled personnel are limited. Such options include bispectral index (BIS) [33], 8 channel Headset-type continuous video EEG monitoring (HS-cv EEG monitoring) [34], and Ceribell [35]. The latter device records limited montage circumferential EEG signals which are converted to sound by a proprietary algorithm. Rapid, simple application of such devices and interpretation at bedside by personnel without EEG training can offer immediate diagnostic information until standard CEEG recording is available.

\section{Treatment}

Identification and treatment of the cause of seizure is as necessary as AEDs for control of ICU seizures. Some seizures are purely symptomatic of underlying medical conditions (e.g., non-ketotic hyperglycemia), medication toxicity, or withdrawal, and so do not require ongoing AEDs. Medications commonly prescribed in the ICU decrease the threshold for seizures, particularly 
antibiotics [36] and psychoactive drugs [37]. Seizures resulting from abrupt withdrawal of $\mathrm{BDZ}$ or AEDs (even when used for non-epilepsy diagnoses) are similar to those arising from withdrawal from recreational drugs and alcohol in that seizures emerging days after ICU admission [38].

Patients with acute intracranial pathology such as parenchymal or subarachnoid hemorrhages [39], meningitis/encephalitis [40], or intracranial masses [41] usually receive AEDs to avert rather than treat seizures. It is common for patients who have cranial neurosurgical procedures to be placed on AED prophylaxis as the risk of immediate post-operative seizures increases the risk of morbidity [42]. This is typically initiated in without any prior EEG testing. Patients who have multiple post-operative seizures, however, may be placed on AED for a more extended period of time beyond the ICU stay. Patients with encephalopathy due to multiple or undetermined etiologies may be suspected of having subclinical seizures, warranting EEG testing to determine the need for AED. Classen, et al. found that $19 \%$ of comatose ICU patients had recorded seizures, $88 \%$ of these within the first $24 \mathrm{~h}$ of monitoring [43].

Patients who are admitted to the ICU with known prior epilepsy may simply require continuation of antiepilepsy drugs (AEDs), but conversion to parenteral agents may be necessary due to ventilatory support, or gastrointestinal limitations. This may necessitate changing the patient from their usually prescribed AED, which may be only available in an oral formulation. Selection of appropriate changes to therapy, especially in an acutely ill patient, requires extensive knowledge of AED drug interactions, side effects, and spectrum of action among seizure types. Patients managed by neurostimulation with vagus nerve stimulator (VNS), responsive neurostimulator (RNS), and deep brain stimulator (DBS), may benefit from programming new device settings. These devices should at least be interrogated to assess battery life and functionality as part of ICU seizure management. Any medially intractable epilepsy patient who is admitted to the ICU is at risk for an exacerbation of epilepsy, with an increased need for supplemental AED or parenteral agents.

Finally, there is a group of patients who are hospitalized in the ICU due to active or impending status epilepticus (SE). These patients may initially present with obvious convulsive activity, but with time convert to a state of electro-mechanical dissociation, with seizures only evident on EEG. NCSE has unclear but high prevalence in comatose patients [44]. Careful application of EEG criteria (Salzburg Consensus Criteria for NCSE) has demonstrated validity in diagnosing NCSE especially with application of the critical care terminology of the American Clinical Neurophysiology Society [45].

Refractory SE(RSE) is defined as SE unresponsive to benzodiazepines (BDZ) and an appropriate second-line AED. RSE develops in 23-43\% of SE cases and leads to even higher morbidity and mortality [46]. New-onset RSE (NORSE) is a rare form of SE often preceded by a clinical prodrome, with half of the cases of undetermined etiology. When a causative illness is identified, the most commonly associated condition is autoimmune encephalitis [47]. Patients who are treated by targeted temperature management (TTM) frequently manifest nonconvulsive or myoclonic seizures during hypothermia or rewarming [48]. Seizures and other malignant EEG patterns, are common following cardiac arrest, with and without TTM, and predict a poor outcome. 
Seizure prophylaxis, while not technically seizure management, is a frequent consideration for ICU patients following severe brain injury, subarachnoid hemorrhage and some intraparenchymal hemorrhages post craniotomy. Historically, phenytoin has been the AED of choice following severe traumatic brain injury [49]. More recent data has placed levetiracetam [50] as a preferred agent not only due to similar efficacy with fewer adverse effects, but also because of potentially improved later outcomes [51]. The Brain Trauma Foundation guideline still recommends phenytoin for the prevention of early post-traumatic seizures ( $<7$ days), but not for the prevention of later seizures or post-traumatic epilepsy. This guideline reports insufficient evidence for recommendation of levetiracetam or other AEDs over phenytoin for early seizure prevention [52]. A possible concern with early use of levetiracetam is the potential for medication-related agitation [53], which may also be an accompaniment of brain injury. Patients who have clinical seizures following craniotomy may be placed on AEDs for a more extended period of time beyond the ICU stay, raising questions of an appropriate duration of longterm AED management.

The initial treatment of an ICU patient presenting with seizures is not very different from less critical seizure patients. If the seizure is isolated and self-limited, only supportive care may be needed. Stabilization of airway, oxygenation, and correction of metabolic derangements may be sufficient, or single doses of benzodiazepines may be used. ICU patients, however, have a lower threshold for seizure due to rapidly changing metabolic states, sleep deprivation medications, and physiologic stress. If clinical seizures are multiple or associated with clear epileptiform patterns on EEG, maintenance medication should be considered.

ICU patients with preserved GU tract function and a feeding tube can often receive their usual oral AEDs by crushing tablets or using alternate enteral formulations of the same AED. Extended release formulations cannot be crushed and must be converted to standard release formulations with dosing interval shortened appropriately. This conversion dose from extended release to standard formulation is 1:1 for all AEDs except valproate (VPA). This extended release formulation is less bioavailable, thus conversion to standard oral forms requires reduction in total daily mg [54]. Liquid VPA (Depakene) more likely causes increased GI distress, so conversion from oral enteric coated tablets of VPA to IV formulation may be preferable. Administering VPA in the tube by opening the "sprinkle" formulation is an alternative, but as this capsule is only available as $125 \mathrm{mg}$ size, larger doses can be inconvenient or difficult to pass through a feeding tube. Oral phenytoin absorption is decreased when administered along with enteral tube feeding, making parenteral use of phenytoin, or preferably fosphenytoin, optimal in patients with feeding tubes [55]. Table 1 lists oral AEDs and formulations suitable for administration by feeding tubes and IV [56].

Patients who are admitted to the ICU and are unable to take their usual home medications due to intubation or enteral restrictions require conversion to a parenteral formulation. If any of these AEDs are unavailable in an IV form, an alternative IV agent must be selected. Review of prior seizure and AED history is helpful in optimizing decisions about alternate agents. If no such history is available, selecting a broad-spectrum parenteral agent with 


\begin{tabular}{|c|c|c|c|}
\hline & $\begin{array}{l}\text { Not compatible with } \\
\text { feeding tube }\end{array}$ & $\begin{array}{l}\text { Oral forms compatible with } \\
\text { feeding tube }\end{array}$ & IV form \\
\hline Brivaracetam & - & Oral solution & Yes \\
\hline Cannabidiol & - & Oral solution & No \\
\hline Carbamazepine & ER & $\begin{array}{l}\text { Chewable } \\
\text { Oral suspension }\end{array}$ & No longer available \\
\hline Clobazam & - & Oral suspension & Substitute IV BDZ \\
\hline Cenobamate & - & Tablet & No \\
\hline Eslicarbazepine & - & Tablet & No \\
\hline Ethosuximide & - & Oral solution & No \\
\hline Felbamate & - & Oral suspension & No \\
\hline Gabapentin & - & Oral solution & No \\
\hline Lacosamide & - & Oral solution & Yes \\
\hline Lamotrigine & $\mathrm{XR}$ & Chewable, ODT & No \\
\hline Levetiracetam & ER & Oral solution & Yes \\
\hline Methsuximide & - & Oral solution & No \\
\hline 0xcarbazepine & ER & Oral suspension & No \\
\hline Perampanel & - & Oral solution & No \\
\hline Phenobarbital & & Elixir & Yes \\
\hline Phenytoin & ER & Oral suspension & $\begin{array}{l}\text { Both phenytoin and fospheny- } \\
\text { toin }\end{array}$ \\
\hline Pregabalin & - & Oral solution & No \\
\hline Primidone & - & Tablet & $\begin{array}{l}\text { No } \\
\text { Metabolized to phenobarbital }\end{array}$ \\
\hline Rufinamide & - & Suspension & No \\
\hline Tiagabine & - & Tablet & No \\
\hline Topiramate & ER & Sprinkle & No \\
\hline Valproate & ER & $\begin{array}{l}\text { Sprinkle } \\
\text { Oral solution }\end{array}$ & Yes \\
\hline Vigabatrin & - & Powder for oral solution & No \\
\hline Zonisamide & - & Open capsule & No \\
\hline
\end{tabular}

minimal side effects and interactions such as levetiracetam is often reasonable. Decision to give a loading dose is based on the clinical acuity of the seizures as well as risk of excess sedation with higher doses.

Seizures which are prolonged or recurrent for at least $5 \mathrm{~min}$ indicate progression to SE and there is urgency to administer effective meds [57]. SE that persists for at least $30 \mathrm{~min}$ has an increased risk of morbidity and mortality. Both the American Epilepsy Society and Neurocritical Care Society SE management guidelines recommend initial treatment with a benzodiazepine followed by a loading dose of a first-line agent $[2,3]$. Prospective trials have shown delays in diagnosis and treatment of SE not only worsen success of SE treatment but mortality [57]. Underdosing of AED during SE is felt to be a contributing factor in unsuccessful treatment [5]. 


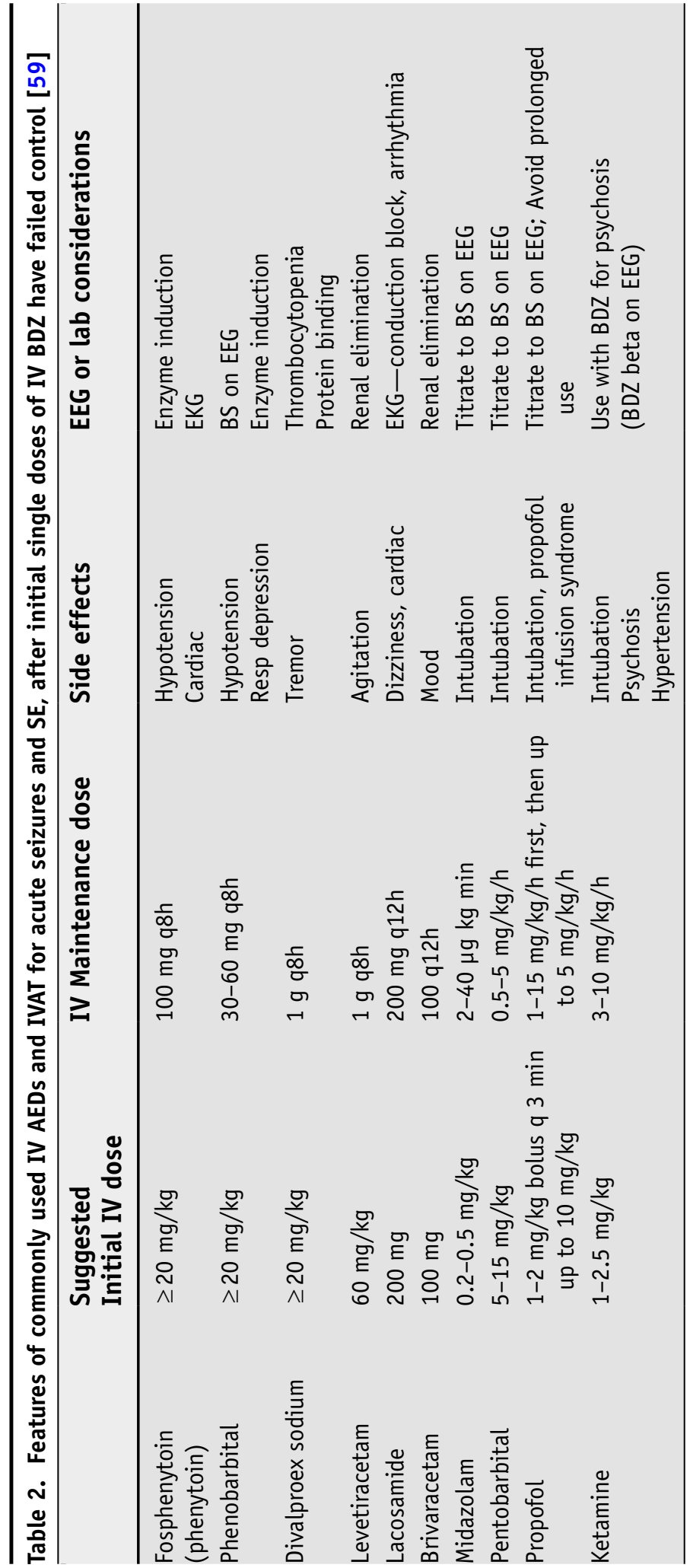


Treatment for SE should take into consideration how administered agents can result in medical complications or interfere with the EEG. BDZ that are given unnecessarily after resolution of a suspected seizure, can result in beta obscuring diagnostic patterns for prolonged periods. Second-line AED selection is often by physician preference, but can be best chosen by considering pharmacokinetics and potential adverse events. In the presence of significant cardiac disease, agents that affect cardiac conduction (e.g., fosphenytoin/phenytoin) or affect cardiac failure (e.g., lacosamide) are less preferable. Poor renal function does not preclude the use of renally eliminated AEDs (e.g., levetiracetam), but dosing interval should be lengthened and lower doses may be necessary. Hepatic dysfunction causes potent enzyme-inducing AEDs (e.g., fosphenytoin/ phenytoin, phenobarbital) to be at higher risk for worsening liver metabolism. VPA, as an enzyme inhibitor, is not contraindicated in hepatic disease, but has highly variable protein binding especially in the ICU patient, and can result in a moderate decrease in platelets as serum levels increase [58].

With failure of the second line AED, SE is considered a refractory and requires selection of a third-line agent, often as IV anesthetic therapy (IVAT). The American Epilepsy Society Treatments Committee recently published a comprehensive review of all parenteral medications including IVAT in convulsive RSE [60]. Table 2 lists commonly used IV AEDs including IVAT.

CEEG is needed during IVAT to ensure maintenance of EEG suppression or burst suppression (BS). Although traditionally 10-s interburst intervals have been recommended, there is no consistent evidence that any interburst interval or burst characteristic predicts seizure resolution on weaning [60]. Prolonged IVAT risks metabolic consequences which may result in permanent cerebral injury. There is insufficient evidence to recommend a specific duration of BS, but prolonged periods during which only pharmacologic BS appears on EEG may conceal accumulating brain injury [61, 62]. Weaning to $50 \%$ of the current IVAT rate every 3-4 h allows for evaluation of the brain function as well as to determine if seizures have recurred. This is optimally coordinated in real time between the ICU team and electroencephalopgrapher. If no seizures recur, weaning should proceed steadily over the next 12-24 h, assessing the EEG for recurrence as dose is lowered. The success of weaning IVAT is dependent on having adequate maintenance AED when dose reduction begins. Slow weaning of IVAT ( $>24 \mathrm{~h}$ ) has not been shown to have lower morbidity or higher risk for seizure recurrence than faster weaning ( $<24 \mathrm{~h}$ ). CEEG should be continued for $24 \mathrm{~h}$ after SE resolves.

\section{Conclusion}

Management in the ICU of seizures, including SE, is made more complicated by multiple medical conditions and polypharmacy in this population. Averting seizure recurrence is difficult due to rapidly changing patient metabolism associated with ICU diagnoses and treatment. Current availability of real-time review of video EEGs as well as access to data in the EMR allows for rapid response and efficient development of management plans. CCEEG can enable early identification of seizures and prioritizes sections of multiple studies for 
detailed review. Limiting factors, however, are resource availability and difficulties achieving efficient and productive communication among members of the ICU team and neurophysiologists.

Although the ICU is the most technologically advanced area of the hospital, seizures in this setting remain complex to diagnose and treat. Redundant and inconclusive diagnostic testing can result from limited historical information and prompt presumptive, butat times, unnecessary treatments. CCEEG can yield either consistent or controversial results, and optimal use of EEG in the ICU requires both use of clear terminology and confirmed understanding of interpretations. In order to efficiently manage of seizures in the ICU, both the treating team and neurophysiologists must maintain clear communication as the clinical and CEEG results rapidly change. Unrealistic expectations by the ICU team for nearly continuous electroencephalopgrapher review and reporting are countered by equally unrealistic expectations by the neurophysiologist to be informed by the ICU team of all suspected seizures and interventions. Such ideal expectations are unfeasible not only due to the complexity of the clinical presentation of seizures, but also due to misconceptions about capabilities within the limitations of staffing, equipment, time, and competing clinical demands. Supplemental technology can fill in some gaps, but the sensitivity and specificity of bedside devices must be clearly understood and supplemented by raw data review by qualified personnel. The ideal (and impossible) situation for managing seizures in the ICU would provide unlimited CEEG resources, staff, and neurophysiologists who never look away from the CEEG, with constant real-time feedback from the ICU team. Even if such an imaginary perfect scenario could be achieved, clinical and EEG correlations would remain difficult. The realistic approach to seizures in the ICU, however, can be optimized by appropriate utilization of CEEG and substitute technology, efficient dosing of AEDs, and weaning of IVAT, with mutually agreeable and reasonable expectations for communication between neurophysiologists and ICU team.

\section{Compliance with Ethical Standards}

\section{Conflict of Interest}

Jane G. Boggs declare no potential conflict of interest.

Human and Animal Rights and Informed Consent

This article does not contain any studies with human or animal subjects performed by the author.

\section{References}

1. Varelas PN, Spanaki MV, Mirski MA. Seizures and the neurosurgical intensive care unit. Neurosurg Clin N Am 2013: Jul;24(3):393-406. 10.1016/j. nec.2013.03.005.
2. Glauser T, Shinnar S, Gloss D, Alldredge B, Arya R, Bainbridge J, Bare M, Bleck T, Dodson WE, Garrity L, Jagoda A, Lowenstein D, Pellock J, Riviello J, Sloan E, Treiman DM. Evidence-Based Guideline: Treatment 
of Convulsive Status Epilepticus in Children and Adults: Report of the Guideline Committee of the American Epilepsy Society. Epilepsy Curr. 2016 JanFeb;16(1):48-61. doi: https://doi.org/10.5698/15357597-16.1.48.

3. Brophy GM, Bell R, Claassen J, Alldredge B, Bleck TP, Glauser T, Laroche SM, Riviello JJ Jr, Shutter L, Sperling MR, Treiman DM, Vespa PM; Neurocritical Care Society Status Epilepticus Guideline Writing Committee. Guidelines for the evaluation and management of status epilepticus. Neurocrit Care. 2012 Aug;17(1):3-23. doi: https://doi.org/10.1007/ s12028-012-9695-z.

4. Trinka E, Cock H, Hesdorffer D, Rossetti AO, Scheffer IE, Shinnar S, Shorvon S, Lowenstein DH. A definition and classification of status epilepticus-Report of the ILAE Task Force on Classification of Status Epilepticus. Epilepsia. 2015;56(10):1515-23. https:// doi.org/10.1111/epi.13121.

5. Sathe AG, Tillman H, Coles LD, Elm JJ, Silbergleit R, Chamberlain J, Kapur J, Cock HR, Fountain NB, Shinnar S, Lowenstein DH, Conwit RA, Bleck TP, Cloyd JC. Underdosing of Benzodiazepines in Patients With Status Epilepticus Enrolled in Established Status Epilepticus Treatment Trial. Acad Emerg Med. 2019;26(8):940-3. https://doi.org/10. 1111/acem.13811.

6. Dzienis W, Tarasów E, Kochanowicz J, Szulc A, Walecki J, Kubas B. Utility of computed tomography and selected MR sequences in the diagnostics of patients with partial epileptic attacks. Med Sci Monit. 2007;13(Suppl 1):49-54.

7. Schultz TL. Technical tips: MRI compatible EEG electrodes: advantages, disadvantages, and financial feasibility in a clinical setting. Neurodiagn J. 2012;52(1):69-81.

8. Benbadis SR, Chen S, Melo M. What's shaking in the ICU? The differential diagnosis of seizures in the intensive care setting. Epilepsia. 2010;51(11):2338-40. https://doi.org/10.1111/j.1528-1167.2010.02683.x.

9. Seneviratne U, Low ZM, Low ZX, Hehir A, Paramaswaran S, Foong M, Ma H, Phan TG. Medical health care utilization cost of patients presenting with psychogenic nonepileptic seizures. Epilepsia. 2019;60(2):349-57. https://doi.org/10.1111/epi. 14625.

10. Viarasilpa T, Panyavachiraporn N, Osman G, Akioyamen NO, Wasade VS, Barkley G, Mayer SA. Intubation for Psychogenic Non-Epileptic Attacks: Frequency, Risk Factors, and Impact on Outcome. Seizure. 2019;31(76):17-21. https://doi.org/10. 1016/j.seizure.2019.12.025.

11. Odak M, Douedi S, Upadhyaya V, Fadhel M, Cosentino J. Focal Neurological Seizure due to Hyperglycemic Hyperosmolar Non-Ketotic Syndrome in Undiagnosed Diabetes Mellitus. Cureus. 2020;12(8): e9909. https:// doi.org/10.7759/cureus.9909.
12. Pellinen J, Carroll E, Friedman D, Boffa M, Dugan P, Friedman DE, Gazzola D, Jongeling A, Rodriguez AJ, Holmes M. Continuous EEG findings in patients with COVID-19 infection admitted to a New York academic hospital system. Epilepsia. 2020. https:// doi.org/10.1111/epi.16667.

13. Pottkämper JCM, Hofmeijer J, van Waarde JA, van Putten MJAM. The postictal state - What do we know? Epilepsia. 2020;61(6):1045-61. https://doi. org/10.1111/epi.16519.

14. Alvarez V, Rossetti AO. Clinical Use of EEG in the ICU: Technical Setting. J Clin Neurophysiol. 2015;32(6):481-5. https://doi.org/10.1097/WNP. 0000000000000194.

15. Sinha SR, Sullivan L, Sabau D, San-Juan D, Dombrowski KE, Halford JJ, Hani AJ, Drislane FW, Stecker MM. American Clinical Neurophysiology Society Guideline 1: Minimum Technical Requirements for Performing Clinical Electroencephalography. J Clin Neurophysiol. 2016 Aug;33(4):303-7. https://doi.org/10.1097/WNP.00000 00000000308

16. ASET Position Statement on Video EEG Data Management in Long Term Monitoring Studies. Neurodiagn J. 2016;56(4):301-306. doi: https://doi.org/10. 1080/21646821.2016.1257340.

17. Gélisse P, Rossetti AO, Genton P, Crespel A, Kaplan PW. How to carry out and interpret EEG recordings in COVID-19 patients in ICU? Clin Neurophysiol. 2020;131(8):2023-31. https://doi.org/10.1016/j. clinph.2020.05.006.

18. Sutter R, Stevens RD, Kaplan PW. Continuous electroencephalographic monitoring in critically ill patients: indications, limitations, and strategies. Crit Care Med. 2013;41(4):1124-32. https://doi.org/10. 1097/CCM.0b013e318275882f.

19. Rossetti AO, Schindler K, Sutter R, Rüegg S, Zubler F, Novy J, Oddo M, Warpelin-Decrausaz L, Alvarez V. Continuous vs Routine Electroencephalogram in Critically Ill Adults With Altered Consciousness and No Recent Seizure: A Multicenter Randomized Clinical Trial. JAMA Neurol. 2020;77(10):1-8. https:// doi.org/10.1001/jamaneurol.2020.2264.

20. Amorim E, Gilmore EJ, Abend NS, Hahn CD, Gaspard N, Herman ST, Hirsch LJ, Lee JW, Cash SS, Westover MB. EEG Reactivity Evaluation Practices for Adult and Pediatric Hypoxic-Ischemic Coma Prognostication in North America. J Clin Neurophysiol. 2018;35(6):510-4. https://doi.org/10.1097/WNP. 0000000000000517.

21. Trinka E, Leitinger M. Which EEG patterns in coma are nonconvulsive status epilepticus? Epilepsy Behav. 2015;49:203-22. https://doi.org/10.1016/j.yebeh. 2015.05 .005$.

22. Osman GM, Araújo DF, Maciel CB. Ictal Interictal Continuum Patterns. Curr Treat Options Neurol. 2018;20(5):15. https://doi.org/10.1007/ s11940-018-0500-y. 
23. Yoo JY, Rampal N, Petroff OA, Hirsch LJ, Gaspard N. Brief potentially ictal rhythmic discharges in critically ill adults. JAMA Neurol. 2014;71(4):454-62. https://doi.org/10.1001/jamaneurol.2013.6238.

24. Rodriguez Ruiz A, Vlachy J, Lee JW, Gilmore EJ, Ayer T, Haider HA, Gaspard N, Ehrenberg JA, Tolchin B, Fantaneanu TA, Fernandez A, Hirsch LJ, LaRoche S; Critical Care EEG Monitoring Research Consortium. Association of Periodic and Rhythmic Electroencephalographic Patterns With Seizures in Critically Ill Patients. JAMA Neurol. 2017 Feb 1;74(2):181188. https://doi.org/10.1001/jamaneurol.2016.4990. The CCEMRC evaluated risk of seizure based on other EEG patterns

25. Struck AF, Tabaeizadeh M, Schmitt SE, Ruiz AR, Swisher CB, Subramaniam T, Hernandez C, Kaleem S, Haider HA, Cissé AF, Dhakar MB, Hirsch LJ, Rosenthal ES, Zafar SF, Gaspard N, Westover MB. Assessment of the Validity of the 2HELPS2B Score for Inpatient Seizure Risk Prediction. JAMA Neurol. 2020;77(4):500-7.

26. Rubinos C, Reynolds AS, Claassen J. The Ictal-Interictal Continuum: To Treat or Not to Treat (and How)? Neurocrit Care. 2018;29(1):3-8. https://doi.org/10.1007/ s12028-017-0477-5.Importantdiscussionofthepotent ialimplicationsofcontroversyofwhethersomeEEGpatter nsareictal.

27. Hopp JL, Sanchez A, Krumholz A, Hart G, Barry E. Nonconvulsive status epilepticus: value of a benzodiazepine trial for predicting outcomes. Neurologist. 2011;17(6):325-9. https://doi.org/10.1097/NRL. 0b013e31822f688c.

28. Ritzenthaler T, Laurencin C, André Obadia N, Bodonian C, Dailler F. Not everything that shakes is a seizure... Role of continuous EEG in the intensive care unit. Neurophysiol Clin. 2017;47(1):13-8. https://doi. org/10.1016/j.neucli.2016.10.001.

29. Sutter R, Ristic A, Rüegg S, Fuhr P. Myoclonus in the critically ill: Diagnosis, management, and clinical impact. Clin Neurophysiol. 2016;127(1):67-80. https://doi.org/10.1016/j.clinph.2015.08.009.

30. Moura LM, Shafi MM, Ng M, Pati S, Cash SS, Cole AJ, Hoch DB, Rosenthal ES, Westover MB. Spectrogram screening of adult EEGs is sensitive and efficient. Neurology. 2014;83(1):56-64. https://doi. org/10.1212/WNL.0000000000000537.

31. Herman ST, Abend NS, Bleck TP, Chapman KE, Drislane FW, Emerson RG, Gerard EE, Hahn CD, Husain AM, Kaplan PW, LaRoche SM, Nuwer MR, Quigg M, Riviello JJ, Schmitt SE, Simmons LA, Tsuchida TN, Hirsch LJ; Critical Care Continuous EEG Task Force of the American Clinical Neurophysiology Society. Consensus statement on continuous EEG in critically ill adults and children, part I: indications. J Clin Neurophysiol. 2015 Apr;32(2):87-95. https://doi.org/10.1097/WNP.0000000000000166.

32. Herman ST, Abend NS, Bleck TP, Chapman KE, Drislane FW, Emerson RG, Gerard EE, Hahn CD, Husain AM, Kaplan PW, LaRoche SM, Nuwer MR, Quigg M, Riviello JJ, Schmitt SE, Simmons LA, Tsuchida TN, Hirsch LJ; Critical Care Continuous EEG Task Force of the American
Clinical Neurophysiology Society. Consensus statement on continuous EEG in critically ill adults and children, part II: personnel, technical specifications, and clinical practice. J Clin Neurophysiol. 2015 Apr;32(2):96-108. https://doi.org/10.1097/WNP.0000000000000165.

33. Ntahe A. Early Diagnosis of Nonconvulsive Status Epilepticus Recurrence with Raw EEG of a Bispectral Index Monitor. Case Rep Crit Care. 2018;12(2018):1208401. https://doi.org/10.1155/2018/1208401.Erratum.In: CaseRepCritCare.2018Dec16;2018:7147626.

34. Egawa S, Hifumi T, Nakamoto H, Kuroda Y, Kubota Y. Diagnostic Reliability of Headset-Type Continuous Video EEG Monitoring for Detection of ICU Patterns and NCSE in Patients with Altered Mental Status with Unknown Etiology. Neurocrit Care. 2020;32(1):217-25. https://doi.org/10.1007/ s12028-019-00863-9.

35. Kamousi B, Grant AM, Bachelder B, Yi J, Hajinoroozi $\mathrm{M}$, Woo R. Comparing the quality of signals recorded with a rapid response EEG and conventional clinical EEG systems. Clin Neurophysiol Pract. 2019;13(4):6975. https://doi.org/10.1016/j.cnp.2019.02.002.Compa risonofutilityofoneofthemorerecentbedsidemethodsfo restimatingEEGpatternswithmoreconventionalCEEG. Wanleenuwat P, Suntharampillai N, Iwanowski P. Antibiotic-induced epileptic seizures: mechanisms of action and clinical considerations. Seizure. 2020;81:167-74.

37. Habibi M, Hart F, Bainbridge J. The Impact of Psychoactive Drugs on Seizures and Antiepileptic Drugs. Curr Neurol Neurosci Rep. 2016;16(8):71. https:// doi.org/10.1007/s11910-016-0670-5.

38. Leach JP, Mohanraj R, Borland W. Alcohol and drugs in epilepsy: pathophysiology, presentation, possibilities, and prevention. Epilepsia. 2012;53(Suppl 4):48-57. https://doi.org/10.1111/j.1528-1167.2012. 03613.x.

39. Feng R, Mascitelli J, Chartrain AG, Margetis K, Mocco J. Anti-epileptic Drug (AED) Use in Subarachnoid Hemorrhage (SAH) and Intracranial Hemorrhage (ICH). Curr Pharm Des. 2017;23(42):6446-53. https://doi.org/10.2174/13816128236661710310 95452.

40. Vezzani A, Fujinami RS, White HS, Preux PM, Blümcke I, Sander JW, Löscher W. Infections, inflammation and epilepsy. Acta Neuropathol. 2016;131(2):211-34. https://doi.org/10.1007/ s00401-015-1481-5.

41. Dewan MC, Thompson RC, Kalkanis SN, Barker FG 2nd, Hadjipanayis CG. Prophylactic antiepileptic drug administration following brain tumor resection: results of a recent AANS/CNS Section on Tumors survey. J Neurosurg. 2017;126(6):1772-8. https://doi.org/10.3171/2016.4.JNS16245.

42. Greenhalgh J, Weston J, Dundar Y, Nevitt SJ, Marson AG. Antiepileptic drugs as prophylaxis for postcraniotomy seizures. Cochrane Database Syst Rev. 2018 May 23;5(5):CD007286. doi: https://doi.org/10. $1002 / 14651858$. 
43. Claassen J, Mayer SA, Kowalski RG, Emerson RG, Hirsch LJ. Detection of electrographic seizures with continuous EEG monitoring in critically ill patients. Neurology. 2004;62(10):1743-8. https://doi.org/10. 1212/01.wnl.0000125184.88797.62.

44. Leitinger M, Beniczky S, Rohracher A, Gardella E, Kalss G, Qerama E, Höfler J, Hess Lindberg-Larsen A, Kuchukhidze G, Dobesberger J, Langthaler PB, Trinka E. Salzburg Consensus Criteria for Non-Convulsive Status Epilepticus-approach to clinical application. Epilepsy Behav. 2015;49:158-63. https://doi.org/10. 1016/j.yebeh.2015.05.007.

45. Krogstad MH, Høgenhaven H, Beier CP, Krøigård T. Nonconvulsive Status Epilepticus: Validating the Salzburg Criteria Against an Expert EEG Examiner. J Clin Neurophysiol. 2019;36(2):141-5. https://doi. org/10.1097/WNP.0000000000000556.Howcareful useofclearterminologycanassistinclarifyingwhichpat ientsareinNCSE.

46. Holtkamp M, Othman J, Buchheim K, Meierkord H. Predictors and prognosis of refractory status epilepticus treated in a neurological intensive care unit. J Neurol Neurosurg Psychiatry. 2005;76(4):534-9. https://doi.org/10.1136/jnnp.2004.041947.

47. Sculier C, Gaspard N. New onset refractory status epilepticus (NORSE). Seizure. 2019;68:72-8. https:// doi.org/10.1016/j.seizure.2018.09.018.

48. Lamartine Monteiro M, Taccone FS, Depondt C, Lamanna I, Gaspard N, Ligot N, Mavroudakis N, Naeije G, Vincent JL, Legros B. The Prognostic Value of 48-h Continuous EEG During Therapeutic Hypothermia After Cardiac Arrest. Neurocrit Care. 2016;24(2):153-62. https://doi.org/10.1007/ s12028-015-0215-9.

49. Temkin NR, Dikmen SS, Wilensky AJ, Keihm J, Chabal S, Winn HR. A randomized, double-blind study of phenytoin for the prevention of post-traumatic seizures. N Engl J Med. 1990;323(8):497-502. https:// doi.org/10.1056/NEJM199008233230801.

50. Brigo F, Bragazzi N, Nardone R, Trinka E. Direct and indirect comparison meta-analysis of levetiracetam versus phenytoin or valproate for convulsive status epilepticus. Epilepsy Behav. 2016;64(Pt A):110-5. https://doi.org/10.1016/j.yebeh.2016.09.030.

51. Bakr A, Belli A. A systematic review of levetiracetam versus phenytoin in the prevention of late post-traumatic seizures and survey of UK neurosurgical prescribing practice of antiepileptic medication in acute traumatic brain injury. Br J Neurosurg. 2018;32(3):237-44. https://doi.org/10.1080/02688697.2018.1464118.

52. Carney N, Totten AM, O'Reilly C, Ullman JS, Hawryluk GW, Bell MJ, Bratton SL, Chesnut R, Harris OA, Kissoon N, Rubiano AM, Shutter L, Tasker RC, Vavilala MS, Wilberger J, Wright DW, Ghajar J. Guidelines for the Management of Severe Traumatic Brain Injury. Fourth
Edition Neurosurgery. 2017;80(1):6-15. https://doi. org/10.1227/NEU.0000000000001432.

53. Chen B, Choi H, Hirsch LJ, Katz A, Legge A, Buchsbaum R, Detyniecki K. Psychiatric and behavioral side effects of antiepileptic drugs in adults with epilepsy. Epilepsy Behav. 2017;76:24-31. https://doi.org/10.1016/j.yebeh. 2017.08.039.

54. Dutta S, Zhang Y. Bioavailability of divalproex extended-release formulation relative to the divalproex delayed-release formulation. Biopharm Drug Dispos. 2004;25(8):345-52. https://doi.org/10.1002/bdd.420.

55. Bauer LA. Interference of oral phenytoin absorption by continuous nasogastric feedings. Neurology. 1982;32(5):570-2. https://doi.org/10.1212/wnl. 32.5.570.

56. Vossler DG, Weingarten M, Gidal BE; American Epilepsy Society Treatments Committee. Summary of Antiepileptic Drugs Available in the United States of America: WORKING TOWARD A WORLD WITHOUT EPILEPSY. Epilepsy Curr. 2018 Jul-Aug;18(4 Suppl 1):1-26. https://doi.org/10.5698/1535-7597. 18.4 s1.1 Comprehensive tabulation of current medications for epilepsy

57. Kellinghaus C, Rossetti AO, Trinka E, Lang N, May TW, Unterberger I, Rüegg S, Sutter R, Strzelczyk A, Tilz C, Uzelac Z, Rosenow F. Factors predicting cessation of status epilepticus in clinical practice: Data from a prospective observational registry (SENSE). Ann Neurol. 2019;85(3):421-32. https://doi.org/10. 1002/ana.25416.

58. Riker RR, Gagnon DJ, Hatton C, May T, Seder DB, Stokem K, Fraser GL. Valproate Protein Binding Is Highly Variable in ICU Patients and Not Predicted by Total Serum Concentrations: A Case Series and Literature Review. Pharmacotherapy. 2017;37(4):500-8. https://doi.org/10.1002/phar. 1912.

59. Vossler DG, Bainbridge JL, Boggs JG, Novotny EJ, Loddenkemper T, Faught E, Amengual-Gual M, Fischer SN, Gloss DS, Olson DM, Towne AR, Naritoku D, Welty TE. Treatment of Refractory Convulsive Status Epilepticus: A Comprehensive Review by the American Epilepsy Society Treatments Committee. Epilepsy Curr. 2020;20(5):245-64. https://doi.org/10.1177/ 1535759720928269.Comprehensiveevidencebase dreviewofparenteralmedicationavailableforRSE.

60. Johnson EL, Martinez NC, Ritzl EK. EEG Characteristics of Successful Burst Suppression for Refractory Status Epilepticus. Neurocrit Care. 2016;25(3):407-14. https://doi.org/10.1007/s12028-016-0294-2.

61. Prisco L, Ganau M, Aurangzeb S, Moswela O, Hallett C, Raby S, Fitzgibbon K, Kearns C, Sen A. A pragmatic approach to intravenous anaesthetics and electroencephalographic endpoints for the 
treatment of refractory and super-refractory status epilepticus in critical care. Seizure. 2020;75:15364. https://doi.org/10.1016/j.seizure.2019.09.011. DiscussionofgoalsofBSwithIVAT.

62. Muhlhofer WG, Layfield S, Lowenstein D, Lin CP, Johnson RD, Saini S, Szaflarski JP. Duration of therapeutic coma and outcome of refractory status epilepticus. Epilepsia. 2019;60(5):921-34. https://doi.org/10.1111/ epi.14706.

\section{Publisher's Note}

Springer Nature remains neutral with regard to jurisdictional claims in published maps and institutional affiliations. 